\title{
Critical Assessment of the Current WADA Approach for the Detection of 4-Chlorodehydromethyltestosteron
}

\author{
Arthur T. Kopylov \\ Institute of Biomedical Chemistry, Moscow, Russia \\ Email: a.t.kopylov@gmail.com
}

How to cite this paper: Kopylov, A.T. (2016) Critical Assessment of the Current WADA Approach for the Detection of 4-Chlorodehydromethyltestosteron. Journal of Analytical Sciences, Methods and Instrumentation, 6, 65-82.

http://dx.doi.org/10.4236/jasmi.2016.64008

Received: December 14, 2016

Accepted: December 27, 2016

Published: December 30, 2016

Copyright $\odot 2016$ by author and Scientific Research Publishing Inc. This work is licensed under the Creative Commons Attribution International License (CC BY 4.0).

http://creativecommons.org/licenses/by/4.0/

\section{(c) (i) Open Access}

\begin{abstract}
This paper is focused on application of method for detection of novel metabolites of 4-chlorodehydromethyltestosterone (known as oral turinabol, OT), which were postulated as long-term metabolites. The method started to be applied without rigorous validation, verification and excretion assay assisted as evidences for existence of the declared detectable compounds as true metabolites derived after OT biotransformation. This method has been started to use almost in its original form which arose in 2012 year for the very first time and never been revised and re-confirmed in peer-reviewed journals. Usually WADA encourages accredited laboratories to publish their results of methods development, validation and specific excretion studies in peer-reviewed journals. However, an incorrect method with neglected validation design and implicit data contradictions is currently widely applied. The author of this presented paper was athletes' representative on repeated occasion and has been provided with several full documentation packages of OT novel metabolites analysis. Thus, the author makes its own conclusion based on its own evidences of that currently employed method and data analysis is exactly the same declared in a wrong way as emerged from the original paper.
\end{abstract}

\section{Keywords}

Anti-Doping, Mass Spectrometry, Substance Identification, Data Analysis, 4-Chlorodehydromethyltestosterone, Turinabol

\section{Introduction}

In summer of 2016 year WADA initiated re-tests of urinary samples obtained 
from the Olympic Games 2008 and 2012. The re-tests aimed to survey the newly discovered metabolites of turinabol (chemical name: 4-chlorodehydromethyltestosterone, CID ref. number 98521), which are characterized as long-term compounds retained in human body for up to 45 - 50 days after administration of turinabol. These proposed long-term metabolites are 4-chloro-17 $\alpha$-methyl$5 \beta$-androstan-3 $\alpha, 16,17 \beta$-triol (M1), 4-chloro-18-nor-17 $\beta$-hydroxymethyl,17 $\alpha$ methyl-5 $\beta$-androsta-1,13-dien-3 $\alpha$-ol (M2), 4-chloro-18-nor-17 $\beta$-hydroxymethyl, $17 \alpha$-methyl-5 $\beta$-androst-13-en-3 $\alpha$-ol (M3) and 4-chloro-18-nor-17 $\beta$-hydroxymethyl,17 $\alpha$-methylandrosta-4,13-dien-3 $\alpha$-ol (M4) (Figure 1). Such properties allow probably unmasking those athletes which avoided punishment due to imperfection of previously employed analytical approach based on the detection of short-term metabolites. Several professional athletes over the world were banned or suspended due to the presence of the prohibited substance of winners of the Olympic Games. Almost all of these athletes have never been tested as positive before. However, rules violation left them only very strict margin of possible actions. If test of the sample has been performed according to International Standards for Laboratories (ISL), the only way for athlete is to demonstrate that WADA accredited laboratories made a significant departure from the ISL and that this departure reasonably caused erroneous adverse analytical finding (AAF). Basically, WADA inclines to believe that result of a test was conducted in a regular manner since the method applied for analysis was perfect and impeccable. Nevertheless, there was an extremely severe point of view in Nature [1] journal delivering shortages in WADA's guidelines for prohibited substances identification criteria. This critical paper opened a few significant problems in data analysis and decision criteria accomplished by scientist associated with WADA. The main conclusion was accommodated in "Nature believes that accepting legal limits of specific metabolites without such rigorous verification

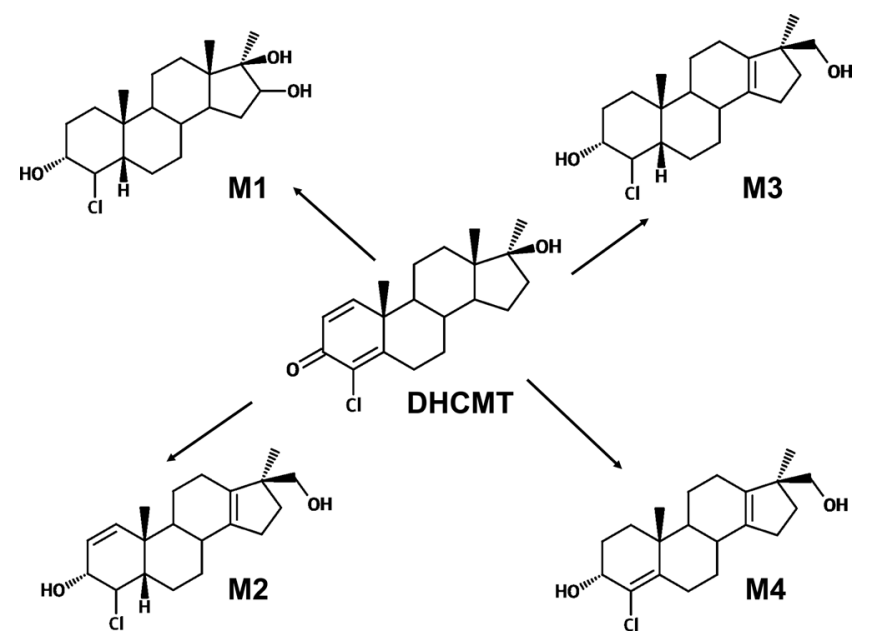

Figure 1. Structure of 4-chlorodehydromethyltestosterone (DHCMT) and proposed structures of long-term metabolites (M1, M2, M3 and M4) of DHCMT. Assumed structure of metabolites is reproduced from the manuscript [2]. 
goes against the foundational standards of modern science, and results in an arbitrary test for which the rate of false positive and false negatives can never be known" [1].

The newly discovered stable metabolites of turinabol were declared for the very first time in manuscript entitled "Detection and mass spectrometric characterization of novel long-term dehydrochloromethyltestosterone metabolites in human urine", which was published in 2012 in Journal of Steroid Biochemistry and Molecular Biology by Tim Sobolevsky and Grigory Rodchenkov [2]. Among the proposed metabolite, the M3 (Figure 1) was the most promising one and depicted broad detection window as assumed. The method used by WADA laboratories for detection of these metabolites is based exactly on this publication. For the past four years, since the manuscript has been published until nowadays, it gained totally 25 citations in different journals [3]. As demonstrated on the SCOPUS preview page [3], most of the authors citing the manuscript are affiliated with WADA laboratories or institutes in one way or another. Only small portion of authors are not related to WADA, which implies that interest in the provided information outcomes from the WADA-affiliated researchers preferably, although anabolic steroids are extensively investigated in cell signaling, drugs development, inhibitory activity, etc. Apparently, this information is not as intriguing for other researchers as expected. It could be seen that the context of citing this manuscript is restricted to referring on either derivatization procedure [4] [5] or strategies for research completely different steroids in blood or urinary matrices [6] [7]. Therefore, the proposed method and observed metabolites may accommodate questions for their validity.

Specifically, it should be noted that WADA technical Document TD2015IDCR [8] (which is actual document when all analysis were being made) requires three diagnostic ions to be used. However, as we will see later, the mass shift for most of ions is $-90 \mathrm{u}$ or $-103 \mathrm{u}$ which corresponds to loss of TMS (trimethylsilanol) or $\mathrm{CH}_{2} \mathrm{O}$ (TMS) from polyhydroxylated steroids with vicinal or primary hydroxyl group, respectively. It should be specifically pointed that these ions do not provide much diagnostics information; notwithstanding, there is nothing in WADA's technical documentation to prevent the analysis from using such ions. A precedent of employment invaluable diagnostic ions (elimination of methyl group, $\left[\mathrm{M}-\mathrm{CH}_{3}\right]^{+}$) has already been in WADA method development story and related to detection of 3'-OH-stanozolol molecule [9]. That time WADA again allows itself to relax identification criteria. Hence, the ions used for discrimination of turinabol-derived molecules do not carry valuable information. Below we will try to review some explicit aspects of the established analytical method and information provided in the manuscript.

\section{Time Elapsed from Discovering of New Metabolites and Absence the Confirmation of Their Existence}

As mentioned before, four years passed from the date of publication and disco- 
vering these new stable metabolites. A good practice in science is developing and confirmation of the finding. From 2012 to 2016 years there was no scientific mentioning about these stable metabolites in any electronic or printed peerreviewed journals. The only referring is in context of common technology of sample preparation and routine processing of urine and blood matrices.

The discovered long-term stable metabolites have never been obtained as pure substance by using organic synthesis, and have never been confirmed by in vitro experiments. The WADA-accredited laboratories up to nowadays use as a standard urine matrices which are "probably" positive for the presence of these metabolites. It still remains as a probability, but not conformity. However, almost all newly discovered biological molecules are used to pass through several validation steps after their primary observation. In this respect, even authors of the established method [2] wrote in "Introduction" section of their manuscript following about previously studied "old" metabolites of turinabol: "In vitro metabolic experiments were also attempted which have shown that the incubation of DHCMT with human cytochrome P450 enzymes resulted in the formation of $6 \beta$-hydroxy metabolite only. And it was not until 2010 when Parr et al. demonstrated the existence of 18-nor-17-hydroxymethyl metabolite of DHCMT, similar to that of methandienone." In this example case, the authors to which referred, demonstrated conformity of the proposed structures by in vitro experiments with cytochrome P450 system. However, no confirmation of the declared structures of stable metabolites was provided for the past years. The same can be said about lack of synthetic standards of the proposed metabolites. For example, when Guddat et al. discovered new metabolites of oxandrolone in 2013, they confirmed the proposed structures via synthesis of these molecules [10] to be sure that they were dealing with correct suggestions.

The discussing method for detection of novel OT metabolites is originally designed and accomplished in incomplete manner. It is hard and time consuming task in metabolomics to discover molecular products of biotransformation. Certainly, preliminary screening approach of all suspicious structures is well applicable. However, the purpose of screening is limitation the number of putative structures. While coming close to the final aim of experiment, strict and sufficient rules should be applied for validation. The results must compliance many analytical characteristics including negative controls, matrix influence factor assay, signal stability, precision, accuracy and, eventually, excretion study. Neither of these features was represented in the discussed method and lack of analytical validity diminishes scientific value to meniscus. The authors [2] developed a new approach using only positive samples from the laboratory collection, and did not provide any evidences for the absence of newly discovered molecules in negative samples. In the "Conclusion" section authors of the novel stable metabolites also suggested, that "The synthesis of reference steroids is needed to confirm the proposed structure of the identified metabolites" [2]. Until today, WADA-ac- 
credited laboratories use only reference urine samples from their collections for the certain analysis. It still happens probably because synthetic references might be a root of contradictions for hypothesis of novel stable metabolites.

More recently, in 2015, Prof. Mario Thevis, who is associated with Cologne Anti-doping Laboratory (Center for Preventive Doping Research, German Sports University in Cologne), published a manuscript about routs of metabolism of turinabol [11]. This is especially interesting point due to the fact that Prof. Thevis and co-authors conducted complete study of OT metabolism by using in vitro whole-cell system of cytochromes $\mathrm{P} 450$. This group of scientists revealed several metabolites of OT, made suggestion of their structures based on massspectrometry data, and confirmed the assumed structures by nuclear magnetic resonance spectroscopy. Another important point in this publication, dedicated to metabolism of turinabol, is lack of mentioning of the stable metabolites discovered in 2012 by Rodchenkov et al. [2], which are currently extensively used by WADA-accredited labs as markers of turinabol ingestion. Instead, Prof. Thevis and co-authors listed several metabolites $(11 \beta-\mathrm{OH}-\mathrm{OT}, 11 \beta, 18$-diOH-OT, $11 \beta$-OH-OT-18-al, 2-OH-OT, 16-OH-OT, and 2,16-diOH-OT) produced by either of cytochromes 11B1, 11B2 and 11A1 [11], but no one of the mentioned metabolites is related to those stated and proposed in 2012 year publication [2] and used by WADA-accredited laboratories. Among other findings, the authors concluded that "these findings suggest that steroidogenic cytochrome P450 enzymes can contribute to drug metabolism and should be considered in drug design and toxicity studies" [11]. Thus, still the metabolism of this prohibited substance is not completely studied.

For years since the publication about newly discovered stable metabolites came out, some sufficient time has passed to provide good experimental evidences for the existence of the proposed molecules. There are numerous possibilities among mentioned above, and one of them is administration of deuterated steroids followed by the identification of the respective deuterated urinary metabolites using hydrogen isotope ratio mass spectrometry (IRMS), as stated, for example, by Dr. Hans Geyer from Center for Preventive Doping Research in Cologne in his publication of 2014 year [5]. So, both WADA-affiliated researchers provided evidences of any newly discovered molecules passing through specifically designed experimental work and routine analysis, in order to convert assumptions into evidence. Any new molecules were recovered and confirmed in many works by (1) in vitro experiments, (2) synthetic substances, (3) other alternative approach, such as NMR spectroscopy, and this line was honored everywhere except for the method discussed. A complete assay of metabolic molecules with rigorous study, excretion assay and structures confirmation, for example androgen receptor modulator, is mandatory practice when findings to be applied in doping control [12]. Nevertheless, no one confirmation experiment has been conducted for all this time. Unfortunately, it leaves the new metabolites 
as an illusion in which merely want to believe. In this way, the discovered structures needed to be re-confirmed because evidences of their existence and their origination from OT are not represented till nowadays. If WADA accredited laboratories do have data of method validation and excretion study, they must represent it in open access peer-reviewed publications.

\section{Lack of Negative Controls and Enormous Number of Candidate Molecules}

The design of experimental workflow for survey and characterization of new stable metabolites of OT consisted of several steps. Initially, the researchers looked for the candidate molecules that probably could be originated from the prohibited substance. For this purpose, the researchers used 10 biological samples (urine samples) available as laboratory reference library [2]. These samples were previously confidently confirmed for the presence of well-known "old" metabolites of turinabol, so-called short-term metabolites. Thus, these samples were characterized as turinabol-positive samples. To simulate the average common metabolome, the researchers polled together aliquots of $10 \mathrm{~mL}$ of each such positive sample and used this mix as a material for presumptive finding of molecules that could be considered as good candidates of turinabol metabolites. No any other negative samples used for control of matrix influence and substance interference factor. In way of deep analysis, the obtained pooled sample was fractionated (after SPE extraction) and fractions were collected under UV-irradiation. As stated, the fractions were collected at stationary detector wavelength of $\lambda=197$ $\mathrm{nm}$. This wavelength is commonly used for steroids due to specific properties allowing these molecules to absorb UV radiation in this range. However, usually during separation and fractionation UV spectra scanning is performed in a wide range of wavelength between $\lambda 200 \mathrm{~nm}$ to $350 \mathrm{~nm}$. It is an important optimization caused by presence of double-bonds, oxo-, hydroxyl groups and halogen functions incorporated in steroidal molecule. The functions and groups significantly increase specificity of UV-based analysis causing appearance of so-called "red-shift" of UV spectra absorbance. A shift in the UV spectra means some physical properties of the sample have changed and this is a signature of electronic transitions assisting in structural determination.

Why this is crucial? Because the proposed molecules of stable metabolites were originated after biotransformation of OT, which is mostly characterized and distinguished from other steroids by presence of a very specific function chlorine $(\mathrm{Cl})$ atom at $\mathrm{C} 4$ position equatorial to $\mathrm{C} 3$-hydroxyl function. Obviously, the chlorine function will not be affected during biotransformation due high toxicity of chlorine for living systems. Notwithstanding, this function can give steric effect of shift the absorbance to about $\Delta \lambda=+30 \mathrm{~nm}$ (bathochromic shift) [13] [14], giving specific signal for isolation and recognition of turinabol-derived molecules. In addition, this data could also be packed as evidence of absence of 
C3-oxo function which would be overshadowed by string absorbance of $\mathrm{C}=\mathrm{C}-\mathrm{C}=\mathrm{O}$ system in A-ring of steroid if it presented [14]. This advantage (presence of chlorine) has not been employed by researchers for the preliminary survey of candidates and routine has not been facilitated. Additionally, presence of double bound $\mathrm{C}=\mathrm{C}$ near to tertiary carbon atom for some metabolites (like M4 and its epi-M4) grants additional advantage for fast recognition by increasing absorbance (hyperchromic effect) in region near to 270 - $280 \mathrm{~nm}$. Unfortunately, no UV spectrum scanning has been performed and only detection at the certain wavelength of $197 \mathrm{~nm}$ was accomplished for fractions collection. Probably, low selectivity of the adjusted UV irradiation detector may be due to the fact of avoidance of possible losses the information during survey procedure, but selective adjustment was enabled. It would be interesting to see such UV spectrum scans for the selected fractions, that contained these stable metabolites, but now it seems impossible. It is merely very convenient to use only single common wavelength of $\lambda=197 \mathrm{~nm}$, which is absolutely unselective and more appropriate in combination with other UV waves, because in this case many questions and doubts cannot be addressed.

This question will turn back our attention further, when we will go to discussion of initial evaluation of GC-MS data. Now, we have to pay attention to one more important thing: the lack of negative controls during method development and validation. Samples free of OT, free of any other prohibited substances, samples obtained after different treatment, or samples obtained after targeted administration any other suspicious substances-these are negative control, which had not been conducted and assayed, as well as blind control experiments had not been provided. More information about possible interference, appearance frequency of the defined stable metabolite, dose-specific appearance should be provided. All these data can be obtained only if correct negative and positive controls would be studied and conducted for analytical assay. Until negative control is conducted, it means that the defined new long-term metabolites can be derived from any other substance at any uncertain conditions and they cannot be strictly attributed to OT only. Some authors' recommendations should also attract an attention of reader: usage of ethyl acetate for liquid-liquid extraction for screening assay then recommendation of usage n-pentane instead for confirmatory assay. However, these solvent are quietly different by their polarity (0.009 for $n$-pentane and 0.228 for ethyl acetate) hence it seems questionable that such different solvent may provide apparently efficient extraction. Substitution with ol- and one-functions probably requires slightly polar solvent rather than pentane.

Any laboratory should seek an appropriate negative and positive control samples especially at the stage of method development and validation to apply further corrective actions for avoidance of interference and investigation of the root of possible unsatisfied events. The negative control samples should be provided 
in the same matrix. Samples of routine laboratory workflow cannot be used for the purpose because (1) analyzed samples are already to be suspicious for the presence and searching of any prohibited substance and (2) these samples are of unknown origination and their "purity" for usage as negative control cannot be confidently appreciated. That should be kept in mind when a laboratory is going to apply a method which has not been validated in a correct way and demonstrates detection of unknown substance of unknown origin. In the presented manuscript [2] devoted to novel stable metabolites investigation and characterization, the negative control samples have not been provided and thus, currently WADA's laboratories use non-validated method for screening and confirmation analysis of basically unspecified molecules that attributed to turinabol metabolites by uncertain reasons. We should mention again the 2015 publication by Prof. Mario Thevis [11], when other metabolites (unrelated to currently used in routine analysis) of turinabol have been obtained in in vitro system, characterized by MS approach and validated by NMR spectroscopy.

When researchers [2] processed the GS-MS data after analysis of the collected fractions, they concluded that 50 metabolite candidates were found in the pooled sample [2]. It is really very questionable because 50 candidates is abnormally big number for the molecules with incorporated chlorine atom. This halogen is very rare in endogenous molecules as well as in xenobiotics. Chlorine is highly toxic and most pharma avoid incorporate it into drug molecules; its presence can be caused by a very specific reasons like in case of turinabol for prevention aromaticity of A-ring of steroidal core. Such enormous number of chlorine-containing candidates suggests that either turinabol is treated by cytochromes P450 in very extensive and complicated ways giving wide range of different metabolites, or that other molecules poorly related to turinabol have also been considered. In case of the first assumption, why only few of candidates were lately chosen as most stable? If so many candidates were initially found, it implies that mostly all of them can be considered as stable metabolites. Currently, it seems that only very convenient candidates that easily can be fitted, or more precisely, can be embedded without suspicion in the lately obtained MS spectra, were chosen. It is really strange finding of so many molecules (50 and 30 after second round of selection) with chlorine because mass spectra of chlorine-containing molecules are very specific and experienced analyst can easily distinguish such compounds. Preselection of not only chlorine containing candidate molecules can be suggested. It is a consequence of initially incorrect adjustments of UV-based fractionation proposed collection of all molecules that can absorb at $\lambda=197 \mathrm{~nm}$, which leads to fictional fractionation step in reality. Because colleting the fractions at poorly specific UV wavelength and across whole analytical time (as stated by authors: in time range $4-30$ minutes; and calculated total time is 30 minutes) results only in reduction the complexity of the matrix, but not preselection of the target compounds or their candidates. 


\section{Allegation about Detection Window of Novel Stable Metabolites}

In the section "Results and discussion", subsection 3.3, Sobolevski et al. wrote: “... the detection window of M3 could be estimated as 40 - 50 days, while M1, M2 and M4 are at least as valuable as I and II" [2]. It is currently known, the metabolite M3 and M2 are most valuable for WADA-accredited laboratories in context of re-analysis of samples for turinabol findings. The majority of new results of re-analysis of 2008 and 2012 years samples were based on adverse analytical findings of metabolite M3 or its epi-mere. In the context of information provided by authors of the analytical method, significant information must be emphasized: “... the detection window of M3 could be estimated as 40 - 50 days ..." [2]. The term "could be estimated" does not mean "validated" or "confirmed". These authors wrote later in the text: "An additional controlled excretion study is needed to fully evaluate the time at which novel metabolites can be detected" [2]. In summary, it is clearly demonstrated that the detection window of 40 - 50 days for so-called novel metabolites is simply assumed to be in so way, and based on the assumption of relative concentration. It is also typical misleading error to suggest the detection window of a new metabolite based on the known data for resemble structure. For example, detection window for betamethasone and its epimere dexamethasone are differed up to 3 times. Only correctly designed excretion assay may provide evidences. There were no any experiments of post-administration analysis to reveal time of biotransformation of turinabol into the certain metabolites, to detect exactly these metabolites in the urine samples after designed administration of turinabol and confirm their detection window, elimination rate as well as structures. We also should mind, that till nowadays there is no any experimental and scientific data regarding to independent observation of these metabolites after administration of turinabol, and no data available regarding real, but not "estimated", observation of detection window of novel metabolites. According to literature search, no one laboratory over the world, even among WADA-associated laboratories [11], has still seen these molecules except for authors. Again, authors stated that additional controlled excretion study is needed, but such study has not been conducted by any independent researcher and by WADA-accredited laboratories, since information of the results is not available anywhere.

It is very dangerous game which some laboratories try to play, because they employ a method which is not validated through specific assay with standards or alternative approaches, not fully controlled due to absence of correct negative control samples collection, not examined on presence of interference from other non-prohibited substances. The method is still not confirmed by specific post-administration excretion assay to reveal the declared metabolites from the living system, in vivo. Eventually, it is confusing that WADA-accredited laboratories rely to half-stuff incomplete method that requires severe and rigorous re- 
vision: found some confusing molecule in probably suspicious samples (that were clear in previous analysis) using non-validated method for detection of unconfirmed metabolite for which detection window was only estimated based on subjective assumption. That is what we get eventually now. The situation bears similarity to the "meldonium saga". After disclosure that the pharmacokinetic data of meldonium elimination and accumulation were obscured, WADA has decided to carry special investigation and research studies, and two best laboratories (in Lausanne and Cologne) were implicated. Apparently, these metabolites need to be taken for careful prior investigation before introducing in routine laboratory screening.

\section{Contradiction of Presented Findings}

Deep study for the details of the method revealed several contradictions of the presented data and their description. We should mention here, that these contradictions are limited by the information provided in the manuscript and a few number of represented spectra. However, some of them are explicit and might shed light on fitting necessary results to spectrum among thousands of them obtained, or vice versa. For the deeper and detailed analysis of the presented data and their believability on corresponding novel metabolites, more data of this experimental workflow is necessary to be analyzed and examined. The provided spectra [2] and experimental data were obtained on GC-MS triple-quadrupole mass spectrometers (QqQ). Obviously, it means that data analysis and processing as well as structures prediction were performed using only low-resolution spectra. It is true, that $\mathrm{QqQ}$ is a conventional way for routine analytical research, but usage of low-resolution and low mass accuracy spectra for spectrum match structures elucidation is absolutely wrongdoing way. The low mass accuracy MS (QqQ) cannot distinguish a very narrow mass difference like, for example, between phosphorylation and sulfonation. Neutral loss of hydrogen chloride can successfully be confused with sequential elimination of hydrogen fluoride (which is included in many pharma) and methyl radical, for example. Thus, obtaining of high resolution spectra of the proposed structures in the isolated fraction would be highly desirable to elucidate full fragmentation spectra and mass accuracy estimation.

We will start from the metabolite M2 (Figure 2) which spectrum is represented on Figure 4 of the manuscript (page 125 of the [2]). This metabolite (M2) is especially discussed by authors due to its high resemblance to another metabolite, M4 (Figure 1). In authors' proposition, their structures differ only by location of double bond in A-ring of steroidal core, which causes spectra similarity for these molecules, however M2 is likely featured by some specific diagnostic ions for its detection and discrimination.

In the text of the manuscript the authors wrote: "At the same time for M2, the ion at $\mathrm{m} / z 377$ sequentially eliminates trimethylsilanol and hydrogen chloride 
followed by the cleavage of the A- and B-rings between C-2/C-3, C-5/C-10 and C-7/C-8 to form the fragments at $m / z$ 287, 251 and 185, respectively" [2]. A closer look on the Figure 2, to which this statement refers, gives us contradictive information (Figure 2).

In the reality, the fragment ion 287 and 251 (Figure 2 and Figure 3) cannot be the products of sequential cleavage of A- and B-ring as postulated by the authors. As shown in the Figure 2, the ion 287 is the product of TMS (trimethylsilanol) group elimination. At this stage of fragmentation, the A-ring still remains intact and no cleavage between $\mathrm{C} 2 / \mathrm{C} 3$ atoms can be inspected. There is additional detail in the spectrum giving evidence that this ion is a product of TMS group loss and this ion cannot appear in spectra after elimination of hydrogen

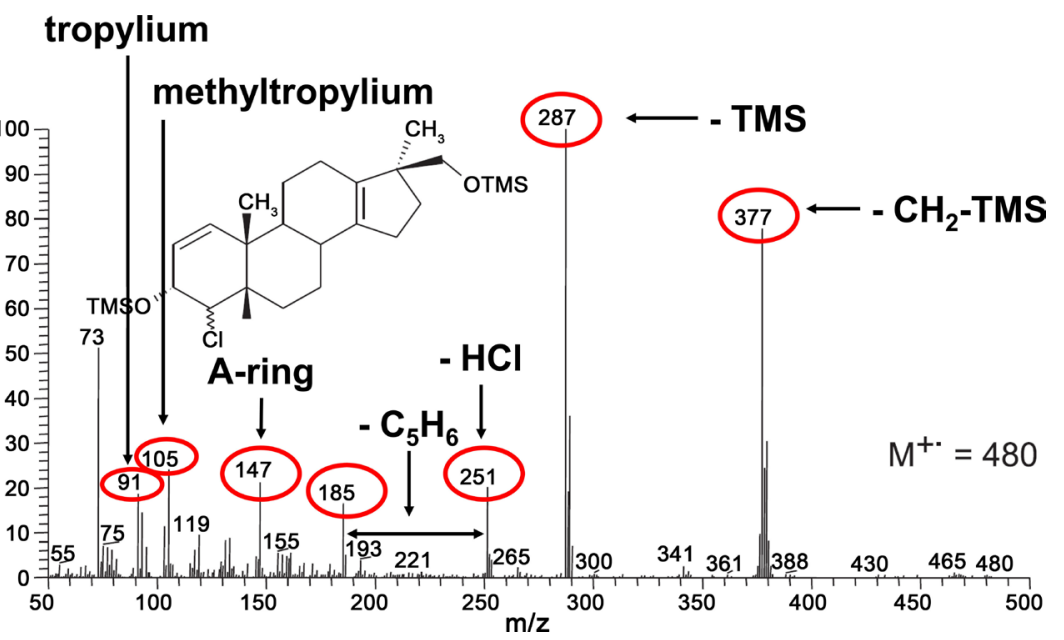

Figure 2. Reproduced figure $4 \mathrm{C}$ of structure and mass spectrum of the pertrimethylsilylated metabolite M2 from original manuscript of Tim Sobolevsky and Grigory Rodchenkov "Detection and mass spectrometric characterization of novel long-term dehydrochloromethyltestosterone metabolites in human urine" in Journal of Steroid Biochemistry \& Molecular Biology 128 (2012) 121-127.

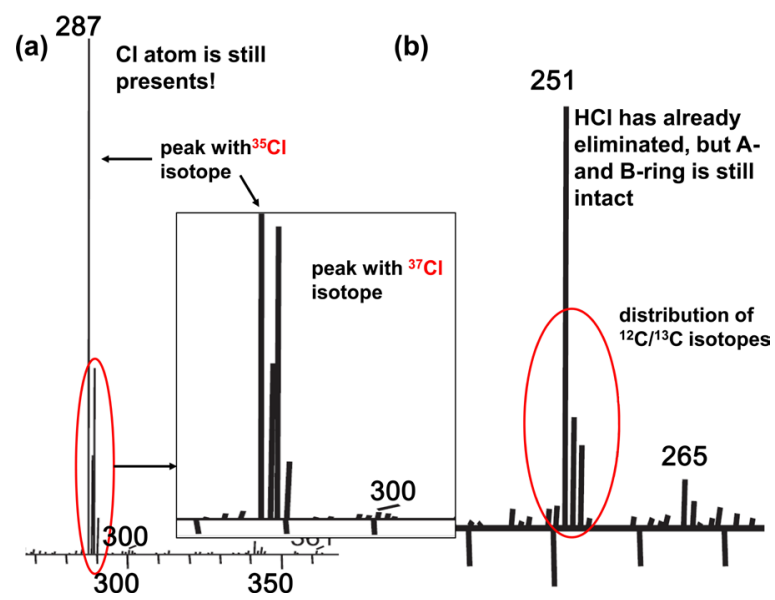

Figure 3. Magnified area of ions located at 287 (a) and 251 (b) of metabolite M2 to demonstrated isotopic distribution with falls in discrepancy with the conclusion of authors. 
chloride: the mass peak of 287 is characterized by isotopic distribution which is highly specific for chlorine atom (Figure 3 ). The chlorine gives main isotopes of 35 and 37 which are stable isotopes and their natural molar fraction input is 0.7576 and 0.2424 , respectively, according IUPAC Technical review for atomic weight of elements [15]. This leads to a conclusion, that the ion 287 still carries chlorine and cannot be produced after A-ring cleavage and loss of hydrogen chloride, as declared by authors. So, the conclusion of the authors goes in conflict with spectrum data.

The ion 251 (Figure 2 and Figure 3) cannot be the product of cleavage A- and B-ring at $\mathrm{C} 2 / \mathrm{C} 3$ and $\mathrm{C} 5 / \mathrm{C} 10$ atoms as well, because this ion is just a product of hydrogen chloride loss. It is clearly demonstrated after detailed investigation of this ion due to absence of chlorine-specific isotopic distribution. Thus, it means that ion 251 is chlorine-deficient ion with intact A- and B-rings, rather than destroyed rings as stated in manuscript.

The ion 185 cannot be the product of cleavage between $\mathrm{C} 7 / \mathrm{C} 8$ as well, because these atoms (C7 and C8) are located in B-ring and the atom C8 is a stacked atom between B- and C-ring. However, by simple calculation it is clearly seen that ion 185 is a product of exactly A-ring cleavage. Even though, origination of this ion still debatable because ion with $\mathrm{m} / \mathrm{z}=185$ is mostly featured for 1-ene-3-keto steroids, however the authors declared that all novel metabolites are $3-\mathrm{OH}$ function and no one has 3-oxo function. So, their conclusions are highly speculative. The most true ion in these spectra of M2, M3 and M4 is ion 147, which is well characterized for 1- or 4-ene steroids, and the ion is displayed everywhere. This is very first ion which is related to exactly A-B-rings cleavage, but which has not been mentioned by authors. Probably, authors did not mentioned about this ion due to considering it as a product of TMS-group migration which is known phenomenon for steroids [16] with more than one TMS-groups and giving an ion with composition $\left[\left(\mathrm{CH}_{3}\right)_{3} \mathrm{SiOSi}\left(\mathrm{CH}_{3}\right)_{2}\right]+$ at $\mathrm{m} / \mathrm{z} 147$. Another two interesting ions in this spectra are ions 91 and 105, which are common steroidal ions called as tropylium cation and methyl tropylium following to the final product of phenyl ion which is not always visible in spectra. These ions (91 and 105) are clearly demonstrated in the spectrum of M2 metabolite, but the question is why only methyl tropylium is visible on the spectra of other investigated metabolites (M3 and M4)?

A conclusion that the defined metabolites do not contain oxo-function due to application of MSTFA/NH4I/DTT gives the same resulting spectra as application of 1\% TCMS (trimethylchlorosilane) is debatable. Griffiths et al. [17] in 2005 reviewed many approaches for chemical derivatization of steroidal molecules for GC-MS purpose, and concluded that composition of MSTFA/NH4I/DTT converts most hydroxyls and ketones in trimethylsylil derivate. Among other scientist, he also referred to publication of Prof. Mario Thevis of 2001 for this conclusion. Regarding TCMS the same authors referred that this chemical con- 
verts some tertiary hydroxyl function and partially oxo-function. The same is postulated by Kasal et al. in "Steroid analysis" book [14]. There are no tertiary hydroxyls in the proposed structures of the novel metabolites, thus identity of the obtained spectra after different chemical derivatization methods can probably be caused by the presence of oxo-function in C3 position. It could be checked by application of chemical approach more specific for oxo-function, which is derivatization by MO (methoxyammonium) to convert mostly oxofunctions into methyl oximes. But this cross-validation has not been performed or maybe not represented by the authors [2] for unknown reasons. Thus, the conclusion of lack the oxo-function in the proposed molecules is not confident, the cross validated experiments with application of more specific for oxo-functions chemistry were not provided by the authors.

Another thought should be turned to suggestion, that for M3 metabolite after loss of hydrogen chloride and both TMS- and $\mathrm{CH}_{2} \mathrm{O}$ (TMS)-groups, the unsaturated A-ring of the steroid preferably has to release butadiene and the product of the retro Diels Alder reaction at $\mathrm{m} / \mathrm{z}$ about 201, and this ion might be one of the most intensive; however, there are no products of the A-ring cleavage which are preferable between $\mathrm{C} 1 / \mathrm{C} 10$ and $\mathrm{C} 4 / \mathrm{C} 5$ atoms for releasing of part of unsaturated ring. Although, the reaction is more preferable for oxo-steroids, but if one refers back to Prof. Thevis publication (2016) [11], which has already been mentioned, his group found at least two metabolites with 16-hydroxy function that can be converted to oxo-function. In this case, the product ion after butadiene loss of unsaturated ring would be at $\mathrm{m} / z$ 217. Nevertheless, we see a very low abundant ion at $m / z$ 183, which can be product of water neutral loss $\left(-\mathrm{H}_{2} \mathrm{O}\right)$ for the eliminated product of retro Diels Alder reaction (Figure 4). This can lead to assumption that the position of moieties in D-ring of M3 could be wrongly suggested and could be in agreement with previously mentioned suggestions of extensive C16 hydroxylation, which consequently prohibits formation of 18-norsteroid if authors wanted to keep molecular weight and structure balanced.

On example of spectrum of metabolite M2 we see valuable ion at $\mathrm{m} / \mathrm{z} 251$. The spectrum of this metabolite has already been discussed earlier and the ion 251 has also been debated. But this ion carries additional important information because it reflects not only ABCD-rings of the compound after loss of hydrogen chloride and both TMS groups as mentioned before, but also the estimated number of double bounds in the remaining ion. Based on the simple calculations it is clearly seen that the ion 251 should reflect presence of four (4) double bonds which seems to be plausible due to unsaturation after neutral losses of TMS moieties and hydrogen chloride (Figure 5). However, additional low abundant ion at 265 reflects possible presence of oxo-function in the remaining ion because oxo-function increases the mass to $14 \mathrm{u}$ in this case $(+16 \mathrm{u}[\mathrm{O}]-2 \mathrm{u}[2 \mathrm{H}])$.

The most important primary information about the structure can be provided by observation of precursor ion, which is $\mathrm{M}^{*}+$, for example, ion 482 for M3 me- 


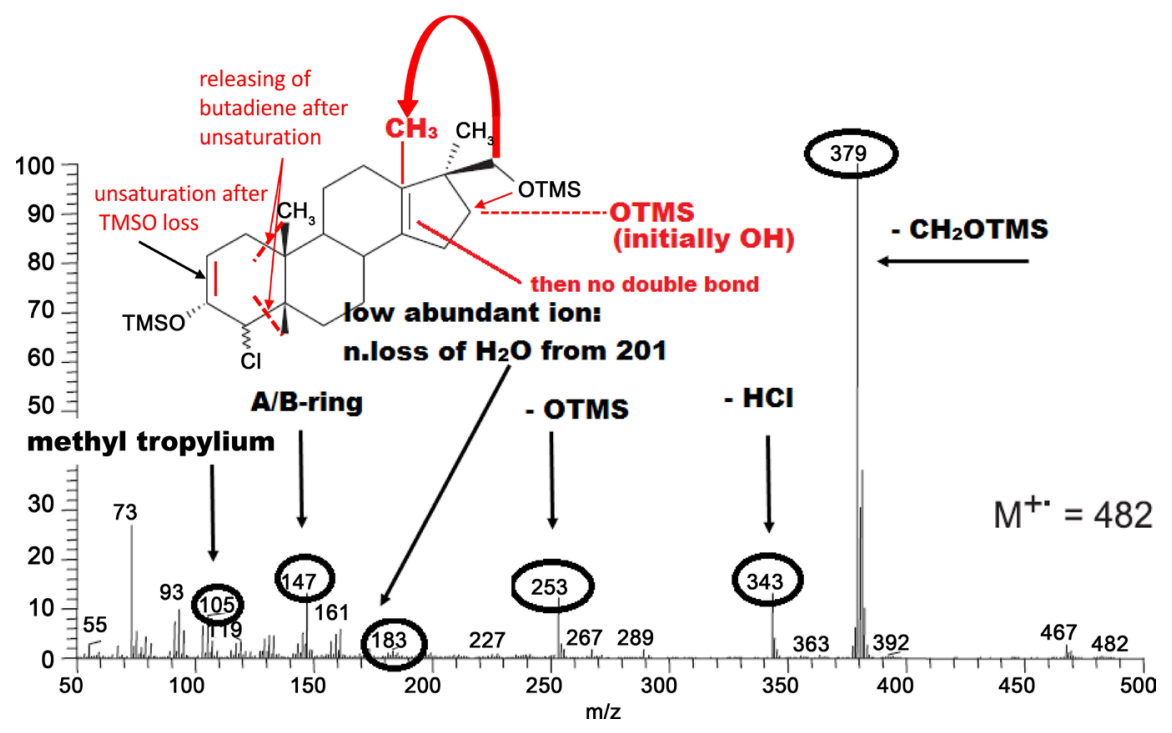

Figure 4. Reproduced figure 4A of the spectrum of metabolite M3 from original manuscript of Tim Sobolevsky and Grigory Rodchenkov "Detection and mass spectrometric characterization of novel long-term dehydrochloromethyltestosterone metabolites in human urine" in Journal of Steroid Biochemistry \& Molecular Biology 128 (2012) 121-127. The image has been modified for this review to show possible wrong interpretation of chemical structure of steroid Ms as well as M2 based on the presented peaks in mass spectrum. The image is commented in the text above. Red lines show probable rearrangements of methyl- and hydroxyl functions.

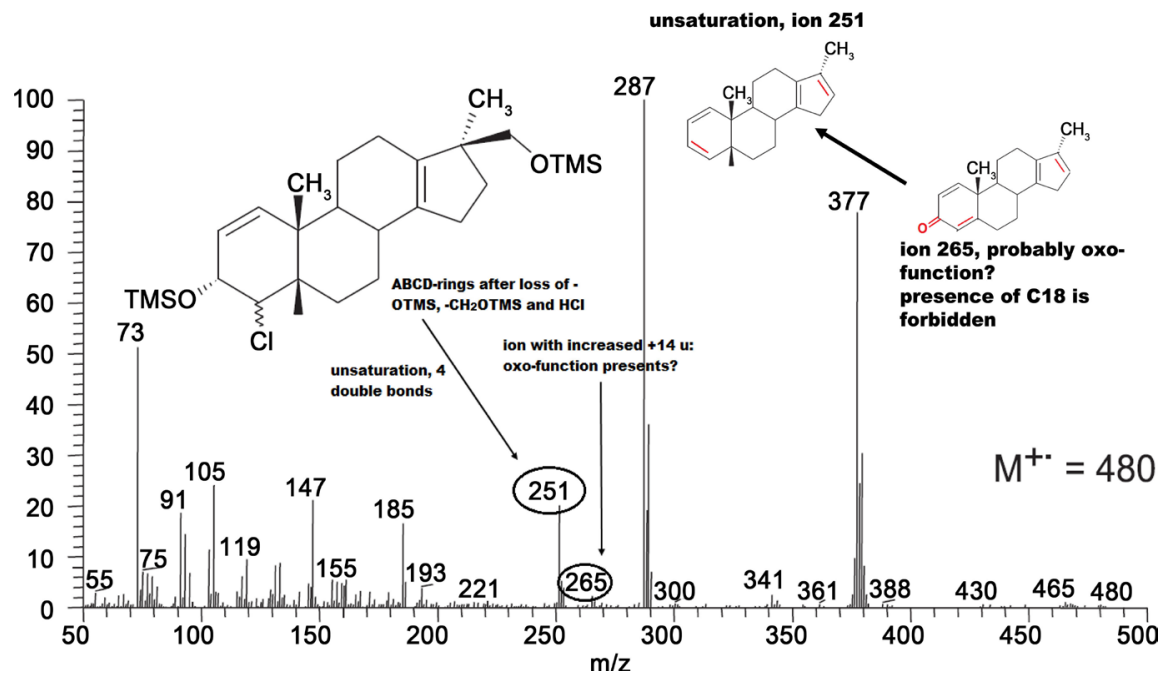

Figure 5. Reproduced figure 4C of mass spectrum of M2 metabolite from original manuscript of Tim Sobolevsky and Grigory Rodchenkov "Detection and mass spectrometric characterization of novel long-term dehydrochloromethyltestosterone metabolites in human urine" in Journal of Steroid Biochemistry \& Molecular Biology 128 (2012) 121-127.

tabolite. But the precursor ions are not shown in the spectra provided for manuscript by some reasons (however, in the same manuscript spectra with existing precursor ion of "old" metabolite I is depicted in the Figure 1 of manuscript 
[2]). Notwithstanding, the ion 379 (after elimination of $\mathrm{CH}_{2} \mathrm{O}$ (TMS)) of $\mathrm{M} 3$ is also suitable to provide some kind of elemental composition. It is known that ions spectra can be used to calculate the number of carbon atoms in molecule. For this purpose we need to know relative abundances of quasi-molecular ion and its first isotope (Figure 6). Specific isotopes distribution due to chlorine atom and input of silicon atom isotopic distribution should be considered in this case. By this calculation we can estimate, that the number of carbon atoms is $(\approx 29 \% / 100 \%) \times 89.9=26.071 \approx 26$ atoms. However, the number of carbon atoms in the proposed structure of metabolite after elimination of $\mathrm{CH}_{2} \mathrm{O}$ (TMS) should contain 22 carbon atoms. To obtain such number $(\mathrm{NC}=22)$ the relative abundance of the first isotope should not exceed $24 \%-25 \%$, thus $(\approx 24.5 \% / 100 \%) \times 89.9=22.0255 \approx 22$ (19 carbon atoms in the steroid molecule and 3 carbon atoms from the remaining TMS group in C3 position, according to the proposed structure). Having the information about calculated number of carbon atoms in the molecule, we can calculate the number of double bonds or rings, or DBE (double bond equivalent) number. This number can be obtained in the following way: $\mathrm{DBE}=\mathrm{X}-(0.5 \mathrm{xY})+(0.5 \mathrm{xZ})+1$, where $\mathrm{X}-$ calculated number of carbon atoms, $\mathrm{Y}$ - number of hydrogen atoms, $\mathrm{Z}$ - number of nitrogen atoms. Due to lack of nitrogen atoms in the proposed molecule, the component $(0.5 \mathrm{xZ})$ shall not be used in calculation. In this way, if we take the $C=22$, which is number of carbons in the proposed molecule, then we get DBE $=4$ (the number of hydrogens in the proposed by authors molecule is $H=38$ ). This number of DBE is true, because there are four rings in the molecule (A-, B-, Cand D-ring), which are typically considered as double bond equivalent. On the

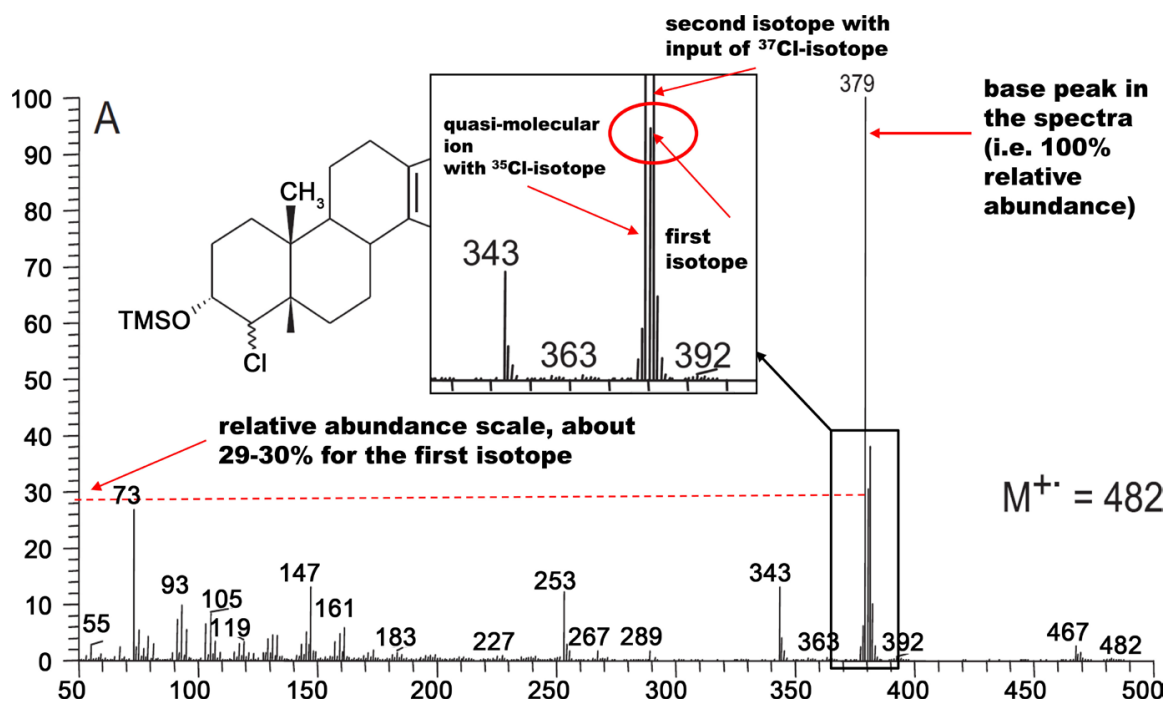

Figure 6. Reproduced figure 4A of mass spectrum of M3 metabolite from original manuscript of Tim Sobolevsky and Grigory Rodchenkov "Detection and mass spectrometric characterization of novel long-term dehydrochloromethyltestosterone metabolites in human urine" in Journal of Steroid Biochemistry \& Molecular Biology 128 (2012) 121-127. 
other hand, if one takes for calculations $C=26$, which is obtained number from the presented spectrum, we will get the final number of $\mathrm{DBE}=8$, which is impossible in this structure even if we consider two double bonds in D-ring of the proposed structure (one originally existed and one derived after unsaturation). It is very questionable: either the structures are impossible, or spectra are not fully related to the proposed structures, but rather fitted to display the steroidal picture.

More details and conclusions can be obtained about the novel metabolites, their confidence and validity of the method. Despite a huge number of discrepancies and remarks found in the basic manuscript by Rodchenkov et al. [2], WADA-accredited laboratories use this method as highly confident and believable in spite of absence of control samples, post-administrative assay, lack of real standards, contradictions to previous and current research and many questionable aspects.

\section{Concluding Remarks}

Doping control as an analytical science always demonstrated a growing interest to accuracy, validity and precision. Actually the method is used by all WADAaccredited laboratories and has been recently employed for re-analyses of samples collected during 2008 and 2012 Olympic Games. Nobody of WADA-affiliated scientists has ever raised the question about validity of the used method; however lots of discrepancies are on the face of the presented material. The authors of "fresh-baked" questionable method know for sure that the scientific community (not only WADA-affiliated) should have been attracted to the discussion, but there was no discussion. There are many challenges of the discussed method that meet such problems as absence of negative controls, confirming absence of interference and side-effect from matrix and other substances, and absence of post-administration assay, confirming that the proposed structures derived from turinabol; therefore the conclusion of detection window for the novel metabolites is invalid.

It is assumed that synthetic standards of the proposed metabolites may enhance (or, in contrary, reduce) the postulated statements in the discussing paper. Special attention should be attracted to inconsistence of the proposed structures with those found and validated by authors already after the publication of the presented method. Although ISL rule does not force laboratories to provide evidences by alternative approaches, but science usually requires confirming and re-confirming. In case of discovery of new substances, confirmation is strictly required instead of just believing. Eventually, huge discrepancies between presented data and its interpretation, and some wrong conclusions arise many questions about whether it has been done intentionally or not. Data analysis and additional data processing is highly desirable. If WADA accredited laboratories do have the internal data of method validity, including excretion study and high- 
resolution full fragmentation spectra, they have to highlight them for open access to provide method integrity. Otherwise, it seems that reputable organization employs mystification instead of science.

These issues are described in our critical review of the method for detection of long-term metabolites of turinabol, and altogether lead to the main conclusion of inability to apply the method in its current state; thus, the results cannot be absolutely believable and require a careful revision by wide scientific community.

\section{Acknowledgements}

Author thanks anonymous athletes and Artem A. Patsev (attorney-at-law) for access to the full Laboratory Documentation Packages, and public non-profit organization "Sport and Law" for support.

\section{Conflict of Interest}

The author declared no conflict of interests.

\section{References}

[1] Editorial (2008) A Level Playing Field? Drug Testing in Sports Aims to Promote Fair, but the Science behind the Tests Needs to Be More Open. Nature, 455, 166.

[2] Sobolevsky, T. and Rodchenkov, G. (2012) Detection and mass Spectrometric Characterization of Novel Long-Term Dehydrochloromethyltestosterone Metabolites in Human Urine. The Journal of Steroid Biochemistry and Molecular Biology, 128, 121-127. https://doi.org/10.1016/j.jsbmb.2011.11.004

[3] SCOPUS Author Preview Source: https://www.scopus.com/results/citedbyresults.uri?sort=plf-f\&cite=2-s2.0-84855679 337\&src=s\&imp=t\&sid=5D49AA58FB0F12DE809BB9F3B00E5228.iqs8TDG0Wy6B URhzD3nFA\%3a130\&sot=cite\&sdt=a\&sl=0\&origin=resultslist\&editSaveSearch=\&t $\underline{\mathrm{xGid}=0}$

[4] Athanasiadou, I., Kiousi, P., Kioukia-Fougia, N., Lyris, E. and Angelis, Y.S. (2015) Current Status and Recent Advantages in Derivatization Procedures in Human Doping Control. Bioanalysis, 7, 2537-2556. https://doi.org/10.4155/bio.15.172

[5] Geyer, H., Schänzer, W. and Thevis, M. (2014) Anabolic Agents: Recent Strategies for Their Detection and Protection from Inadvertent Doping. British Journal of Sports Medicine, 48, 820-826. https://doi.org/10.1136/bjsports-2014-093526

[6] Kiousi, P., Angelis, Y.S., Fragkaki, A.G., Georgakopoulos, C. and Lyris, E. (2015) Markers of Mesterolone Abuse in Sulfate Fraction for Doping Control in Human Urine. Journal of Mass Spectrometry, 50, 1409-1419. https://doi.org/10.1002/jms.3715

[7] Yadav, S.K., Chandra, P., Goyal, R.N. and Shim, Y.-B. (2013) ChromatographyBased Determination of Anabolic Steroids in Biological Fluids: Future Prospects Using Electrochemistry and Miniaturized Microchip Device. Chromatographia, 76, 1439-1448. https://doi.org/10.1007/s10337-012-2351-0

[8] WADA Technical Document-TD2015IDCR (2015) Minimum Criteria for Chromatographic-Mass Spectrometric Confirmation of the Identity of Analytes for 
Doping Control Purposes. Version Number 1.0. http://www.wada-ama.org

[9] Larsson, M. (2011) Substance Identification in Anti-Doping Control by Means of Mass Spectrometry. Data Reduction and Decision Criteria. Journal of Analytical Sciences, Methods and Instrumentation, 1, 19-24. https://doi.org/10.4236/jasmi.2011.12003

[10] Guddat, S., Fußhöller, G., Beuck, S., Schänzer, W. and Thevis, M. (2013) Synthesis, Characterization, and Detection of New Oxandrolone Metabolites as Long-Term Markers in Sports Drug Testing. Analytical and Bioanalytical Chemistry, 405, 82858294. https://doi.org/10.1007/s00216-013-7218-1

[11] Schiffer, L., Brixius-Anderko, S., Hannemann, F., Zapp, J., Neunzig, J., Thevis, M. and Bernhardt, R. (2016) Metabolism of Oral Turinabol by Human Steroid Hormone-Synthesizing Cytochrome P450 Enzymes. Drug Metabolism and Disposition, 44, 227-237. https://doi.org/10.1124/dmd.115.066829

[12] Thevis, M., Thomas, A., Möller, I., Geyer, H., Dalton, J.-T. and Schänzer, W. (2011) Mass Spectrometric Characterization of Urinary Metabolites of the Selective Androgen Receptor Modulator S-22 to Identify Potential Targets for Routine Doping Controls. Rapid Communications in Mass Spectrometry, 25, 2187-2195. https://doi.org/10.1002/rcm.5100

[13] Engel, L.L. (1963) Pure and Applied Biology, Vol. 3: Physical Properties of the Steroid Hormones. Pergamon Press, New York.

[14] Kasal, A., Budesinsky, M. and Griffiths, W.J. (2010) Spectroscopic Methods of Steroid Analysis. Chapter 2: Steroid Analysis. Springer, Berlin.

[15] De Laeter, J.R., Böhlke, J.K., de Bièvre, P., Hidaka, H., Peiser, H.S., Rosman, K.J.R. and Taylor, P.D.D. (2003) Atomic Weights of the Elements: IUPAC Technical Report. Pure and Applied Chemistry, 75, 683-800.

[16] Gustafsson, J.-A., Ryhage, E., Sjövall, J. and Moriarty, R.M. (1969) Migration of the Trimethylsilyl Group upon Electron Impact in Steroids. Journal of the American Chemical Society, 91, 1234-1236. https://doi.org/10.1021/ja01033a045

[17] Griffiths, W.J., Shackleton, C. and Sjövall, J. (2005) Steroid Analysis. In: The Encylopedia of Mass Spectrometry, Vol. 3, Elsevier, Oxford, 447-472.

\section{Scientific Research Publishing}

\section{Submit or recommend next manuscript to SCIRP and we will provide best service for you:}

Accepting pre-submission inquiries through Email, Facebook, LinkedIn, Twitter, etc.

A wide selection of journals (inclusive of 9 subjects, more than 200 journals)

Providing 24-hour high-quality service

User-friendly online submission system

Fair and swift peer-review system

Efficient typesetting and proofreading procedure

Display of the result of downloads and visits, as well as the number of cited articles

Maximum dissemination of your research work

Submit your manuscript at: http://papersubmission.scirp.org/

Or contact jasmi@scirp.org 